\title{
Evolution of Brown Dwarf Atmospheres: Investigating Physical Parameters from Near-IR Spectra
}

\author{
Nadya Gorlova ${ }^{1}$, Michael R. Meyer, Jim Liebert, George H. Rieke \\ Department of Astronomy, The University of Arizona, Tucson, AZ, \\ USA 85721
}

\begin{abstract}
We obtained near-infrared spectra of a sample of very low mass objects as a function of age in order to investigate the temperature and surface gravity sensitivity of several features in the $\mathrm{J}$ - and $\mathrm{K}$-bands.
\end{abstract}

\section{Introduction}

In order to study the initial mass function in young open clusters at the low mass end, one needs to have a fast and reliable way of separating pre-MS members from foreground dwarfs and background giants. These objects occupy same place on the color-magnitude diagram, but have different surface gravities. Using this fact, we decided to explore the gravity-sensitive features in the near-infrared spectra of the objects of spectral types $\mathrm{M}-\mathrm{L}$, corresponding to the range of masses near sub-stellar boundary.

\section{Observations}

Using FSpec IR spectrograph on the 6.5m MMT telescope (Mt. Hopkins, Arizona), we obtained J \& K spectra of $\sim 30$ brown dwarf candidates, SpT M4-L5, drawn from the field and open clusters and associations ( $\rho$ Oph, Tau, IC 348, Upper Sco, Pleiades) to sample a range of gravities $\log g \sim 3$ to 5 dex (Fig.1), as well as 3 late $\mathrm{M}$ giants $(\log g \sim 0)$. The resolution of our smoothed spectra is $\sim 700$ and an average $\mathrm{S} / \mathrm{N}$ in flux is $40-80$ (depending on the feature).

\section{Results}

We calculated gravities using masses from the theoretical $T_{\text {eff }}-L$ diagram, assuming dwarf colors and having distances to most of our objects. The correlation of some features measured from our spectra with gravity is shown in Fig.1.

We confirm strong temperature dependence of $\mathrm{H}_{2} \mathrm{O}$ and $\mathrm{CO}$ bands (e.g.,. Reid et al. 2001) and infer that gravity dependence of these features is insignificant compared to that of $\mathrm{FeH}, \mathrm{KI}$ and $\mathrm{NaI}$ lines. While the weakness of the latter features in cluster compared to field objects was known before (e.g.,.

\footnotetext{
${ }^{1}$ Email: ngorlova@as.arizona.edu
} 
Martín et al. 1996, Luhman et al. 1998, Béjar et al. 1999, Lucas et al. 2001), no systematic investigation of this effect has been carried out so far, especially in the $\mathrm{J}$ band. IR is the only option to work with cool, low mass and embedded objects, and in the $\mathrm{J}$ band the atomic features are especially prominent.

We also compared our spectra to recent atmospheric calculations by Allard et al. 2001 with the conclusion that while they predict correct trends, the systematic offsets between observations and theory remain (Fig.1).

Using our results we managed to confirm low surface gravity of two brown dwarfs from the TW Hya and Taurus associations, whose membership was originally proposed based on their optical spectra (2MASS1139-3159 and CFHTBD-Tau4).
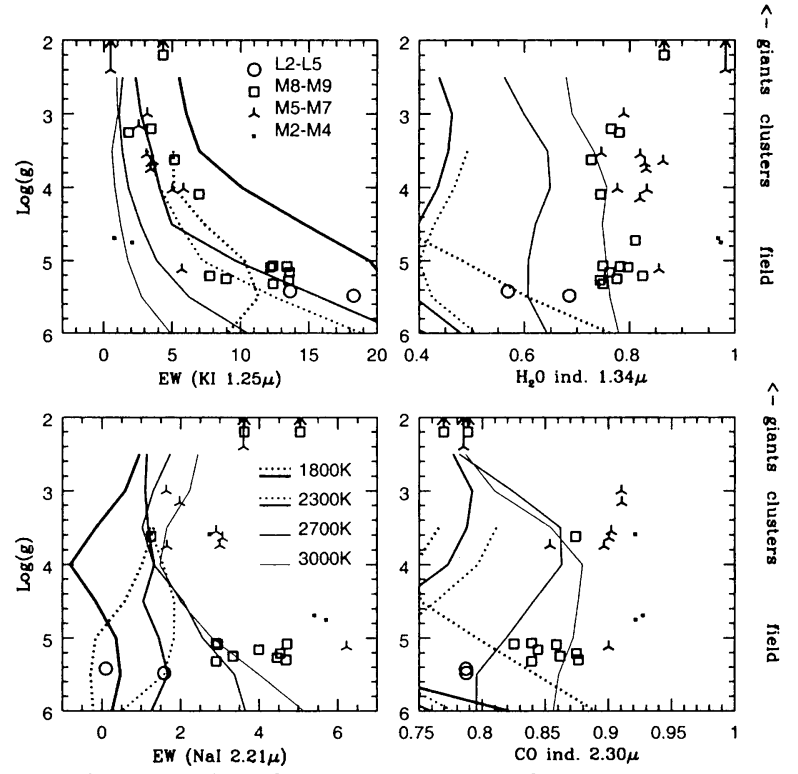

Figure 1. The trends of the strength of some absorption features in the near-IR with gravity and temperature, as measured from our spectra (symbols) and PHOENIX model spectra (dots- Dusty2000 and solid-Cond2000). Giants are indicated by arrows as their surface gravities are off scale on this plot $(\log g \sim 0)$.

\section{References}

Allard, F., Hauschildt, P. H., Alexander, D. R., et al. 2001, ApJ, 556, 357

Béjar, V. J. S., Zapatero Osorio, M. R., \& Rebolo, R., 1999, ApJ, 521, 671

Lucas, P. W., Roche, P. F., Allard, F., \& Hauschildt P. H., 2001, MNRAS, 326, 695

Luhman, K. L., Rieke, G. H., Lada, C. J., \& Lada, E. A. 1998, ApJ, 508, 347

Martín, E. L., Rebolo, R., \& Zapatero Osorio, M. R. 1996, ApJ, 469, 706

Reid, I. N., Burgasser, A.J., Cruz, K. L. et al. 2001, AJ, 121, 1710 